\title{
Effects of salt reduction on cardiovascular risk factors. The STRIVE-study. A 4-month randomized controlled study among healthy families
}

\section{Abstract}

Dietary salt intake in most countries is far beyond the recommended level and many countries have initiated national programs to decrease salt intake. However, potential adverse effects of reducing salt intake in the general population are currently discussed. Hence some randomized studies have shown adverse effects on plasma lipids, the renin-angiotensin-aldosterone system and the sympathetic nervous system, which may again increase risk of CVD. These findings are however mainly based on short-term studies with a very large acute salt reduction. This study aims to explore the effect of gradually reducing salt intake in a real life setting according to recommendations, and to examine the effect of different salt reduction strategies among families.

The study was a 4-month cluster randomized controlled trial with families randomly assigned to either A) salt reduced bread, B) salt reduced bread and dietary counselling or C) standard bread (control) Participants in intervention A received bread gradually reduced in salt content from $1.2 \mathrm{~g}$ salt $/ 100 \mathrm{~g}$ (regular) to $0.6 \mathrm{~g}$ salt $/ 100 \mathrm{~g}$ in ryebread and $0.4 \mathrm{~g} \mathrm{salt} / 100 \mathrm{~g}$ in wheat bread. Participants in intervention $B$ received the same salt reduced bread and in addition, they received dietary advise on how to further reduce their salt intake and promote potassium. The control group received regular bread. Changes in outcomes were assessed using linear mixed models.

Results: A total of 89 families (155 adults; 156 children) from the suburb of Copenhagen, Denmark, participated in the study (February-July 2018). A total of 291 (94\%) participants completed the intervention. Results presented are preliminary.

Intention to treat analyses showed no significant effects of the salt reduction intervention on changes in systolic and diastolic blood pressure, plasma triglyceride. A small, but significant $(-0.26 \mathrm{mmol} / \mathrm{l} ; \mathrm{P}=0.02)$ decrease in total plasma cholesterol was shown in intervention group A. A similar, but non-significant, tendency was found in intervention group B. (more results will follow).

Subgroup analyses, including only participants in the intervention groups that decreased their salt intake by at least $20 \%$ from baseline to 4-month follow-up, showed a significant decrease in diastolic $(-3.5 \mathrm{mmHg} ; \mathrm{P}<0.0001)$ and systolic $(-6.3 \mathrm{mmHg} ; \mathrm{P}<0.0001)$ blood pressure, total plasma cholesterol $(-0.25 \mathrm{mmol} / 1 ; \mathrm{P}=0.0009)$, LDL plasma cholesterol (logarithm transformed) $(-6 \%$; $\mathrm{P}=0.03)$ and plasma triglyceride (logarithm transformed) $(-17 \% ; \mathrm{P}=0.04)$. No significant effects were found for HDL plasma cholesterol, aldosterone, renin, plasma glucose and HbAlc.

Conclusion: Reduced salt intake were associated with beneficial changes in cardiovascular risk factors. No adverse effects were observed.

\section{Conflict of Interest}

There is no conflict of interest 